\title{
Tumor metabolism: metabolic alterations and heterogeneity in cancer progression
}

\author{
Anna Sebestyén ${ }^{1}$ \\ Published online: 16 December 2021 \\ (c) The Author(s), under exclusive licence to Springer Science+Business Media, LLC, part of Springer Nature 2021
}

The number of malignant cells even in a small tumor could be extremely high. According to some estimates, there are more cells in a 10-g tumor than humans alive on the planet. The tumor mass, which contains malignant and surrounding non-malignant cells in the tissue microenvironment, is extremely diverse. The importance of intra-tumoral heterogeneity-highlighted as immunophenotype heterogeneity nearly 40 years ago in Cancer and Metastasis Reviewswas introduced by Miller and Heppner [1-3]. Tumor tissue heterogeneity contributes to the appreciated somatic evolutionary process in cancer development and progression $[4$, 5]. While the elements (mutations, selections, genetic drifts, fitness, epistasis) of this evolutionary process are weakly described, it is conventionally accepted that selection acts on phenotype rather than genotype, which is mainly deterministic. Eliminating such a complex and often disseminated metastatic population such as cancer is more than challenging. The diversity-served signaling, compensatory and adaptation mechanisms in tumor progression could be the basis of therapeutic failures. Gene-sequencing-centric views have dominated molecular cancer research to date, but among genetic diversity and heterogeneity further factors influence the alterations in the tumor cell population. In the last two decades, several phrases provided understanding of the evolutionary and ecologic alterations in tumor development. In addition to genetic diversity, local and time-dependent tissue heterogeneity, immune regulatory differences, tumor tissue symbiosis, plasticity, and metabolic heterogeneity have gained special attention. Metabolic adaptation, symbiosis, plasticity, and fitness have been used to describe tumor evolution. It is well established that accelerated tumor growth can occur only with sufficient energy, building blocks, and

Anna Sebestyén

hsebanna@gmail.com;

sebestyen.anna@med.semmelweis-univ.hu

1 1st Department of Pathology and Experimental Cancer Research, Semmelweis University, Budapest, Hungary nutrient support. Metabolic flexibility and plasticity are necessary for cancer progression. Environmental adaptation, metabolic activity, and rewiring are critical for maintaining the survival and growth of the tumor population [6-8].

Altered metabolism and its potential role in tumor development were described nearly a century ago [9]. Although Nobel Prize winner Otto Warburg spent his entire carrier in cancer metabolism, new scientific research overshadowed his studies of metabolic regulatory failures. It took nearly 100 years to rediscover the significance of his research. Characteristic hallmarks of cancer were completed with several features including metabolic reprogramming by Hanahan and Weinberg 10 years ago [10]. Metabolic plasticity has been highlighted as an important factor in the progression and survival of the tumor cell population [11]. Additionally, recent developments in this research field underline the importance of metabolic heterogeneity and symbiosis mediated adaptation mechanisms in cancer tissues.

The oncogene addiction in tumorigenesis is well known. We can analyze and target these in personalized therapy. However, recent findings and failures in targeted therapies suggest that the study and mapping of the metabolic rewiring in tumor tissues may be necessary to overcome therapy resistance. Developments in biochemistry, molecular biology, and genomics must be applied to understand and clarify the relationship between metabolic networking and genetic regulation in cancer.

In this perspective, the emerging experimental data characterized metabolic differences in normal versus tumor cells and developing technologies/model systems help to define novel targets and find new or current drugs for cancer prevention, early diagnosis, and treatments, and decrease cancer mortality. In this dedicated issue, certain views of tumor metabolism and metabolic heterogeneity have been collected and reviewed in line with recent studies. Hopefully, this issue can attract more attention to previously described and studied aspects of metabolic rewiring in cancer progression and metastasis. 


\section{References}

1. Miller, F. R. (1982). Intratumor immunologic heterogeneity. Cancer Metastasis Reviews, 1(4), 319-334. https://doi.org/10.1007/ bf00124215

2. Heppner, G. H., \& Miller, B. E. (1983). Tumor heterogeneity: Biological implications and therapeutic consequences. Cancer Metastasis Reviews, 2(1), 5-23. https://doi.org/10.1007/bf000 46903

3. Heppner, G. H. (1984). Tumor heterogeneity. Cancer Research, 44(6), 2259-2265.

4. Pogrebniak, K. L., \& Curtis, C. (2018). Harnessing tumor evolution to circumvent resistance. Trends in Genetics, 34(8), 639-651. https://doi.org/10.1016/j.tig.2018.05.007

5. Greaves, M., \& Maley, C. C. (2012). Clonal evolution in cancer. Nature, 481(7381), 306-313. https://doi.org/10.1038/nature10762

6. Martinez-Outschoorn, U. E., Bartrons, M., \& Bartrons, R. (2019). Editorial: Cancer ecosystems. [Editorial]. Frontiers in Oncology, 9, 718. https://doi.org/10.3389/fonc.2019.00718

7. Zahir, N., Sun, R., Gallahan, D., Gatenby, R. A., \& Curtis, C. (2020). Characterizing the ecological and evolutionary dynamics of cancer. Nature Genetics, 52(8), 759-767. https://doi.org/10. 1038/s41588-020-0668-4

8. McGuirk, S., Audet-Delage, Y., \& St-Pierre, J. (2020). Metabolic fitness and plasticity in cancer progression. Trends Cancer, 6(1), 49-61. https://doi.org/10.1016/j.trecan.2019.11.009

9. Warburg, O., \& Minami, S. (1923). Versuche an Überlebendem Carcinom-gewebe. Klinische Wochenschrift, 2(17), 776-777. https://doi.org/10.1007/BF01712130

10. Hanahan, D., \& Weinberg, R. A. (2011). Hallmarks of cancer: The next generation. Cell, 144(5), 646-674. https://doi.org/10.1016/j. cell.2011.02.013

11. Mosier, J. A., Schwager, S. C., Boyajian, D. A., \& Reinhart-King, C. A. (2021). Cancer cell metabolic plasticity in migration and metastasis. Clinical \& Experimental Metastasis, 38(4), 343-359. https://doi.org/10.1007/s10585-021-10102-1

Publisher's note Springer Nature remains neutral with regard to jurisdictional claims in published maps and institutional affiliations. 